\title{
Should endocrine therapy still be used as an initial treatment for postmenopausal women with metastatic breast cancer?
}

\author{
Laura Orlando ${ }^{*, 1} \&$ Saverio Cinieri ${ }^{1}$
}

First draft submitted: 6 March 2017; Accepted for publication: 21 April 2017; Published online: 7 July 2017

Hormonal receptor (HR) positive breast cancer is a heterogeneous disease and accounts for about $70 \%$ of breast cancer. Despite standard adjuvant therapy, over $20 \%$ of HR-positive breast cancer patients experience recurrence, and all patients with metastatic disease succumb to their illnesses [1].

Endocrine therapy is the mainstay of treatment for HR-positive advanced breast cancer (ABC). Guidelines recommend the preferential use of endocrine therapy for $\mathrm{HR}$ positive/HER-2-negative $\mathrm{ABC}$, even in the presence of visceral metastases, and reserve chemotherapy for cases of rapidly progressive disease [2,3]. Usually, the sequential use of different endocrine agents is the preferred option, except for life-threatening diseases [4]. The preferred first-line endocrine therapy depends on the type and duration of previous adjuvant endocrine therapy. Available data support the use of aromatase inhibitors (AI), tamoxifen or fulvestrant $[5,6]$.

Many Phase III studies compared AIs with tamoxifen as the first-line therapy, favoring AIs in terms of progression-free survival (PFS), response rate and clinical benefit rate.

A recent meta-analysis of six trials (2560 patients) comparing an AI with tamoxifen found a significant advantage in response rate and clinical benefit rate for AI [7].

Data from the randomized Phase III trial (NCT01602380) comparing fulvestrant $500 \mathrm{mg}$ to anastrozole for the treatment of HR-positive untreated ABC were published. Fulvestrant demonstrated superiority compared with anastrozole and met its primary end point of extended PFS (16.6 months for fulvestrant, 13.8 months for anastrozole (HR: 0.797) [8].

Even though endocrine therapy is able to achieve disease control and prolonged PFS for HR-positive ABC, endocrine resistance may develop in patients treated with endocrine therapies (acquired resistance) or in untreated patients (de novo resistance). Several efforts have been made to explain and overcome the mechanisms of endocrine resistance and novel therapeutic strategies have been developed.

\section{Mechanisms of resistance \& new}

drugs

Loss of ER expression, ER- $\alpha$ (ESR1) mutation, epigenetic mechanisms regulating ER expression and crosstalk between ER and intracellular signaling pathways are the main mechanisms of resistance to endocrine therapy [9]. The most interesting mechanisms are the upregulation of PI3K/

\section{KEYWORDS}

- endocrine-positive breast cancer

- endocrine resistance

\section{Future Medicine part of}


Akt/mTOR pathway signaling and Cyclin D/ $\mathrm{CDK} 4 / 6 / \mathrm{Rb}$ pathway aberration [10].

The knowledge of these mechanisms has led to the discovery of novel drugs able to contrast aberrant pathways and restore endocrine sensibility.

Rapalogs are among the first agents introduced in the clinic that inhibit the activity of mTOR complex 1. BOLERO-2 trial (NCT00863655), a double-blind Phase III trial that randomly selected 724 postmenopausal women to receive exemestane with everolimus at $10 \mathrm{mg}$ a day or placebo, it showed an advantage in terms of PFS for the combination arm (7.8 months for everolimus compared with 3.2 months for placebo; hazard ratio: 0.45$)$. Median overall survival was not significantly improved (31 months with everolimus compared with 26.6 months with placebo) [11,12].

Based on the results, everolimus is now approved in combination with exemestane for AI-resistant ABC. It should be underlined that about $21 \%$ of population enrolled in BOLERO-2 received the assigned treatment as first-line therapy, after progression to adjuvant therapy with AI.

Regarding first-line therapy for HR-positive $\mathrm{ABC}$, three CDK4/6 inhibitors are in advanced stages of clinical development. Palbociclib and ribociclib received US FDA approval as firstline therapy for $\mathrm{HR}+\mathrm{HER} 2$ negative ABC. PALOMA-1 (NCT00721409) was a Phase II trial that randomized 165 postmenopausal women with untreated $\mathrm{HR}$-positive $\mathrm{ABC}$ to receive letrozole with or without palbociclib [13]. The impressive advantage in PFS with the experimental therapy (20.2 vs 10.2 months (hazard ratio: $0.49 ; \mathrm{p}=0.0004$ ) has led to accelerated approval for palbociclib and letrozole as firstline treatment of postmenopausal patients with $\mathrm{HR}+\mathrm{ABC}$. The randomized Phase III, doubleblind trial PALOMA 2 confirmed these results (PFS: 24.8 months for palbociclib and letrozole vs 14.5 months for placebo and letrozole; hazard ratio: $0.58 ; \mathrm{p}=0.0001$ ) [14].

Data with Ribociclib have been presented at European Society for Medical Oncology (ESMO) meeting for first-line therapy of HR-positive HER2-negative ABC (NCT01958021). In this randomized, placebo-controlled Phase III trial, 668 women with untreated ABC were enrolled. Patients were assigned to receive ribociclib (600 mg per day on a 3 weeks on, 1 week off) plus letrozole or placebo plus letrozole.
Treatment with ribociclib prolonged the PFS rate compared with letrozole alone (63 vs $42 \%$; $\left.\mathrm{p}=3.29 \times 10^{-6}\right)[15]$.

\section{Open questions}

The possibility to overcome endocrine resistance by blocking intracellular pathway has been one of the greater successes in breast cancer treatment. Based on the results of Phase III trials, a new scenario is now raised for firstline therapy of HR-positive population. The attempt to prevent the onset of endocrine resistance will lead to consider the monotherapy with endocrine agents a depowered treatment. But, should all patients with HR-positive HER2 negative $\mathrm{ABC}$ receive a combination therapy upfront? For which patient should endocrine therapy alone be considered an adequate treatment? Which inhibitor and which endocrine agent should be chosen?

Palbociclib and ribociclib showed a quite similar efficacy and a superimposable toxicity profile and both are actually approved as first-line treatment of HR-positive HER2-negative ABC.

The enrolled population in the two trials is similar, but not the same. In PALOMA 2 trial, a greater number of patients recurred early after completion of adjuvant endocrine therapy (about 20\% of patients) [14] compared with MONALEESA 2 trial (1.2\%) [15]. In PALOMA 3, a Phase III, double blind trial (NCT01942135) which compared palbociclib plus fulvestrant versus placebo plus fulvestrant in pretreated $\mathrm{ABC}$ patients, $24.8 \%$ patients recurred during or after adjuvant therapy and received the assigned treatment as first-line therapy. The trial has shown significant improvement in PFS (9.2 vs 3.8 months; hazard ratio: 0.42) [16]. Even though this combination will be approved as therapy for pretreated patients, can we affirm the combination of palbociclib and fulvestrant is not as adequate as first-line therapy for all the patients?

Moreover, can we delay the introduction of an endocrine agent using an anti-CD $4 / 6$ inhibitors alone? Abemaciclib is the only tested drug showing clinical activity as monotherapy in pretreated patients. Results from a Phase II trial of abemaciclib (NCT02102490) in 132 patients with HR positive/HER2 negative pretreated ABC have been recently presented [17]. Median PFS was 5.7 months, clinical benefit $42.4 \%$. Phase III trials are ongoing with abemaciclib monotherapy or in combination as first-line therapy. 


\section{Toxicity}

The safety profile of all these new agents is peculiar and requires appropriate management.

The primary toxicities of everolimus are stomatitis, metabolic disorders (hyperglycemia and hyperlipidemia), noninfectious pneumonitis, immunosuppression, fatigue and anorexia [11].

Palbociclib and ribociclib are CDK4/6 inhibitors, and abemaciclib inhibits CDK4/6/9. The main adverse events of these drugs reflect the different spectra of inhibition. The most common adverse reactions of palboclib and ribociclib were neutropenia, leukopenia, fatigue and anemia $[13,15]$. The most common adverse events attributable to abemaciclib were diarrhea, nausea, fatigue, neutropenia and vomiting [17].

\section{Predictive markers}

Despite the rapid understanding of mechanisms underlying resistance to endocrine therapy has led to clinical development of new target agents, predictive biomarkers helpful to identify those patients who are more likely to respond to these drugs are still needed. To date, markers of PI3K/ AKT/mTOR pathway activation or CDK4/6 pathway aberration (CCND1 or CDK4 amplification, $R B 1$ mutation and $C D K N 1 B$ loss) have not identified a specific population most likely to benefit from these innovative therapies $[18,19]$.

\section{Which therapy at progression?}

The introduction of biological agents in association with endocrine therapy for $\mathrm{ABC}$ raised the question about the effectiveness of following lines of therapy. If perturbation of cellular pathways involved in cell proliferation and survival by CDK4/6 inhibition is able to compromise the efficacy of further hormonal therapies is actually unknown.

All registered clinical trials neither allowed treatment switch onto the experimental arm in case of progression or involved a crossover design upfront. Despite the limits of crossover design, this approach could be used as a test for treatment strategies in HR-positive ABC.

Moreover, it is reasonable to speculate that impressive efficacy and rapid approval of the targeted and endocrine combinations will compromise further development of new drugs with exclusive endocrine activity, in other words, new selective estrogen receptor modulators (Serms) or Selective estrogen receptor degraders (Serds)?

\section{Other combinations}

The cell-cycle arrest induced by CDK4/6 inhibitors might elicit an antagonism if chemotherapy is used concurrently. In retinoblastoma protein positive triple-negative breast cancer cell lines and xenografts, CDK4/6 inhibition and consequent cell-cycle arrest prevented the induction of DNA damage by taxanes and anthracyclines [20]. Moreover, due to the superimposable toxicity, a combination of CDK4/6 inhibitors and cytotoxic drugs may need to be approached with caution, despite the palbociclib-induced bone marrow suppression occurred through cell-cycle arrest and not apoptotic cell death [20]. Actually, trials designed to compare mTOR/PI3K inhibitors or CDK4/6 inhibitors with chemotherapy are ongoing.

\section{Conclusion}

A combination of strategies that target both ER and resistant pathways are therefore rational approaches to overcome endocrine resistance. Toxicity and costs are increased with the addition of targeted agents. There is a critical need to develop predictive biomarkers in order to identify patients more likely to benefit from combination therapies. An accurate evaluation of costs and benefits and a special consideration of the optimal sequential therapeutic strategy is needed in the treatment of HR-positive ABC patients.

\section{Financial \& competing interests disclosure}

The authors have no relevant affliations or financial involvement with any organization or entity with a financial interest in or financial conflict with the subject matter or materials discussed in the manuscript. This includes employment, consultancies, honoraria, stock ownership or options, expert testimony, grants or patents received or pending, or royalties.

No writing assistance was used in the production of this manuscript.

\section{References}

Papers of special note have been highlighted as:

- of interest; $\bullet$ of considerable interest

1 Early Breast Cancer Trialists' Collaborative Group. Aromatase inhibitors versus tamoxifen in early breast cancer: patient-level metaanalysis of the randomized trials. Lancet 386, 1341-1352 (2015).

2 Rugo HS, Rumble RB, Macrae E. Endocrine therapy for hormone receptor-positive metastatic breast cancer: American Society of Clinical Oncology guideline summary. J. Oncol. Pract. 12, 583-587 (2016).

- Highlights the role of multiple lines of endocrine therapy in the therapeutic strategy 
for hormonal receptor positive advanced breast cancer.

3 Cardoso F, Costa A, Norton L et al. ESO-ESMO 2nd international consensus guidelines for advanced breast cancer (ABC2). Ann. Oncol. 23, 489-502 (2014).

4 Perez EA. Treatment strategies for advanced hormone receptor-positive and human epidermal growth factor 2-negative breast cancer: the role of treatment order. Drug Resist. Updat. 24, 13-22 (2016).

5 Gibson L, Lawrence D, Dawson C, Bliss J. Aromatase inhibitors for treatment of advanced breast cancer in postmenopausal women. Cochrane Database Syst. Rev. 4, CD003370 (2009).

6 Di Leo A, Jerusalem G, Petruzelka L et al. Results of the CONFIRM Phase III trial comparing fulvestrant $250 \mathrm{mg}$ with fulvestrant $500 \mathrm{mg}$ in postmenopausal women with estrogen receptor-positive advanced breast cancer. J. Clin. Oncol. 28, 4594-4600 (2010).

7 Xu HB, Liu YJ, Li L. Aromatase inhibitor versus tamoxifen in postmenopausal woman with advanced breast cancer: a literaturebased meta-analysis. Clin. Breast Cancer 11, 246-251 (2011).

8 Robertson JF, Bondarenko IM, Trishkina E et al. Fulvestrant $500 \mathrm{mg}$ versus anastrozole 1 $\mathrm{mg}$ for hormone receptor-positive advanced breast cancer (FALCON): an international, randomized, double-blind, Phase III trial. Lancet 388, 2997-3005 (2016).

9 García-Becerra R, Santos N, Díaz L, Camacho $\mathrm{J}$. Mechanisms of resistance to endocrine therapy in breast cancer: focus on signaling pathways, miRNAs and genetically based resistance. Int. J. Mol. Sci. 14, 108-145 (2012).
- Insights into the issues of different mechanisms of endocrine resistance.

10 Butt AJ, McNeil CM, Musgrove EA, Sutherland RL.Downstream targets of growth factor and oestrogen signalling and endocrine resistance: the potential roles of c-Myc, cyclin D1 and cyclin E. Endocr. Relat. Cancer 12, S47-S59 (2005).

11 Yardley DA, Noguchi S, Pritchard KI et al. Everolimus plus exemestane in postmenopausal patients with $\mathrm{HR}(+)$ breast cancer: BOLERO-2 final progression-free survival analysis. Adv. Ther. 30, 870-884 (2013).

12 Piccart M, Hotobagyi GN, Campone M et al. Everolimus plus exemestane for hormone-receptor-positive, human epidermal growth factor receptor-2-negative advanced breast cancer: overall survival results from BOLERO-2. Ann. Oncol. 25, 2357-2362 (2014).

13 Finn RS, Crown JP, Lang I et al. The cyclin-dependent kinase 4/6 inhibitor palbociclib in combination with letrozole versus letrozole alone as first-line treatment of oestrogen receptor-positive, HER2- negative, advanced breast cancer (PALOMA-1/ TRIO-18): a randomised Phase II study. Lancet Oncol. 16, 25-35 (2015).

- First randomized trial showing the superiority of combination of CDK4/6 inhibitor and endocrine therapy over endocrine therapy alone as first-line therapy in metastatic breast cancer.

14 Finn RS, Martin M, Rugo HS et al. Palbociclib and Letrozole in advanced breast cancer. NEJM 375, 1925-1936 (2016).
15 Hortobagyi GN, Stemmer SM, Burris HA et al. Ribociclib as first-line therapy for HR-positive, advanced breast cancer. $N$. Engl. J. Med. 375, 1738-1748 (2016).

16 Turner NC, Ro J, Andre F et al. Palbociclib in hormone-receptor- positive advanced breast cancer. N. Engl. J. Med. 373, 209-219 (2015).

- First randomized trial showing the superiority of combination of CDK4/6 inhibitor and endocrine therapy over endocrine therapy alone in pretreated metastatic breast cancer.

17 Dickler MN, Tolaney SM, Rugo HS et al. MONARCH1: results from a Phase II study of Abemaciclib, a CDK4 and CDK6 inhibitor, as monotherapy, in patients with $\mathrm{HR}+1$ HER- breast cancer, after chemotherapy for advanced disease. J. Clin. Oncol. 34(Suppl.), Abstr 510 (2016).

18 Hortobagyi GN, Piccart-Gebhart MJ, Burris HA et al. Correlation of molecular alterations with efficacy of everolimus in hormone receptor-positive, HER2-negative advanced breast cancer: results from BOLERO-2. J. Clin. Oncol. 31(Suppl. 26), 142 (2013).

19 Dean JL, McClendon AK, Knudsen ES. Modification of the DNA damage response by therapeutic CDK4/6 inhibition. J. Biol. Chem. 287, 29075-29087 (2012).

$20 \mathrm{Hu}$ W, Sung T, Jessen BA et al. Mechanistic investigation of bone marrow suppression associated with palbociclib and its differentiation from cyctotoxic chemotherapies. Clin. Cancer Res. 22, 2000-2008 (2016). 